\title{
Decidualisation of human endometrial stromal cells is associated with increased expression and secretion of prorenin
}

Eugenie R. Lumbers ${ }^{1}$, Yu Wang ${ }^{1}$, Sarah J. Delforce ${ }^{1}$, Celine Corbisier de Meaultsart ${ }^{1}$, Philip C. Logan²,4, Murray D. Mitchell ${ }^{3}$ and Kirsty G. Pringle ${ }^{1 *}$

\begin{abstract}
Background: In pregnancy, the decidualised endometrium expresses high levels of prorenin and other genes of the renin-angiotensin system (RAS) pathway. In this study we aimed to determined if the RAS was present in endometrial stromal cells and if decidualisation upregulated the expression of prorenin, the prorenin receptor ((P)RR) and associated RAS pathways. Immortalised human endometrial stromal cells (HESCs) can be stimulated to decidualise by combined treatment with medroxyprogesterone acetate (MPA), 17ß-estradiol $\left(E_{2}\right)$ and CAMP (MPAmix) or with 5-aza-2'-deoxycytidine (AZA), a global demethylating agent.
\end{abstract}

Methods: HESCs were incubated for 10 days with one of the following treatments: vehicle, MPA-mix, a combination of medroxyprogesterone acetate (MPA) and estradiol-17 $\beta$ alone, or AZA. Messenger RNA abundance and protein levels of prorenin (REN), the (P)RR (ATP6AP2), angiotensinogen (AGT), angiotensin converting enzyme $(A C E)$, angiotensin II type 1 receptor (AGTR1), vascular endothelial growth factor (VEGF), and plasminogen activator inhibitor-1 (PAI-1) were measured by real-time PCR and ELISA's, respectively. Promyelocytic zinc finger (PLZF) and phospho-inositol-3 kinase (PIK3R1) mRNA abundances were also measured.

Results: HESCs expressed the prorenin receptor (ATP6AP2), REN, AGT, ACE and low levels of AGTR1. MPA-mix and AZA stimulated expression of REN. Prorenin protein secretion was increased in MPA-mix treated HESCs. E 2 + MPA had no effect on any RAS genes. MPA-mix treatment was associated with increased VEGF (VEGFA) and PAI-1 (SERPINE1) mRNA and VEGF protein.

Conclusions: An endometrial prorenin receptor/renin angiotensin system is activated by decidualisation. Since (P)RR is abundant, the increase in prorenin secretion could have stimulated VEGF $A$ and SERPINE1 expression via Ang II, as both ACE and AGTR1 are present, or by Ang II independent pathways. Activation of the RAS in human endometrium with decidualisation, through stimulation of VEGF expression and secretion, could be critical in establishing an adequate blood supply to the developing maternal placental vascular bed.

Keywords: Prorenin, ATP6AP2, Angiotensinogen, Decidualisation, 5-aza-2'deoxycytidine, Angiotensin, VEGF

\footnotetext{
* Correspondence: Kirsty.pringle@newcastle.edu.au

${ }^{1}$ School of Biomedical Sciences and Pharmacy, University of Newcastle,

Newcastle, Australia and Mothers and Babies Research Centre, Hunter

Medical Research Institute, Level 3 East, 1 Kookaburra Circuit, New Lambton

Heights, NSW 2305, Australia

Full list of author information is available at the end of the article
}

(c) 2015 Lumbers et al. Open Access This article is distributed under the terms of the Creative Commons Attribution 4.0 International License (http://creativecommons.org/licenses/by/4.0/), which permits unrestricted use, distribution, and reproduction in any medium, provided you give appropriate credit to the original author(s) and the source, provide a link to the Creative Commons license, and indicate if changes were made. The Creative Commons Public Domain Dedication waiver (http://creativecommons.org/publicdomain/zero/1.0/) applies to the data made available in this article, unless otherwise stated. 


\section{Background}

The human endometrium/decidua expresses all of the components of the renin-angiotensin system (RAS) [1, 2] including the prorenin receptor [3]. Morphological studies show dense expression of the RAS in the uterine glandular epithelium and in endometrial stromal cells. Stromal cells express renin, angiotensin converting enzyme (ACE) and the angiotensin II type $1\left(\mathrm{AT}_{1} \mathrm{R}\right)$ and type $2\left(\mathrm{AT}_{2} \mathrm{R}\right)$ receptors in a cyclical manner, suggesting they are controlled by the sex hormones estrogen and progesterone $[1,2]$.

Recently, we have shown that in late gestation, the decidua, which is the transformed endometrial lining of the pregnant human uterus, expresses prorenin mRNA $(R E N)$ and secretes prorenin $[4,5]$. Interestingly, levels of expression of prorenin in the decidua are influenced by the sex of the fetus [5].

Tissue RASs affect cell growth and proliferation and stimulate angiogenesis. Tissues other than the kidney only secrete prorenin which is inactive and unable to cleave angiotensin I (Ang I) from renin substrate (angiotensinogen, AGT), unless it is activated by proteases, low $\mathrm{pH}$ [6] or cold [7]. Alternatively, prorenin can be activated by binding to the prorenin receptor which allows the pro-segment of the enzyme to unfold, exposing the catalytic site $[8,9]$. The prorenin receptor is identical to the M8.9 segment (ATP6AP2) of vacuolar $\mathrm{H}^{+}$-ATPase (V-ATPase, [9]). Once Ang I is formed, the RAS cascade is initiated, with Ang I conversion to Ang II by angiotensin converting enzyme (ACE, Fig. 1). Ang II is the major biologically active peptide, and via the $A_{1} R$, is responsible for vasoconstriction, aldosterone secretion, angiogenesis and cell proliferation. Acting via its $\mathrm{AT}_{2} \mathrm{R}$ it has actions that generally oppose the effects of the Ang II$\mathrm{AT}_{1} \mathrm{R}$ interaction as does the downstream peptide Ang(1-7) which is formed from Ang II by ACE2.

The prorenin/prorenin receptor interaction not only has the potential to generate Ang peptides in tissues but it has other Ang II-independent actions (Fig. 1). These may be synergistic with Ang II in their effects on angiogenesis, tissue growth and differentiation. These are shown in Fig. 1 and include activation of ERK1/2 and HSP27/p38 signalling pathways, translocation of the transcription factor promyelocytic leukaemia zinc finger protein (PLZF) to the nucleus and subsequent upregulation of the p85 $\alpha$ subunit of PI3kinase, as well as suppression of prorenin receptor gene expression (ATP6AP2, $[8,10,11])$. Other actions of the prorenin/prorenin receptor interaction include maintenance of V-ATPase and activation of Wnt signaling [9, 12]. Since decidualisation involves cell growth and proliferation as well as angiogenesis it is very likely that with decidualisation there is upregulation of prorenin/prorenin receptor pathways that promote angiogenesis and cell proliferation.

A human endometrial stromal cell (HESC) line derived from endometrial stromal cells of uterine myomas can

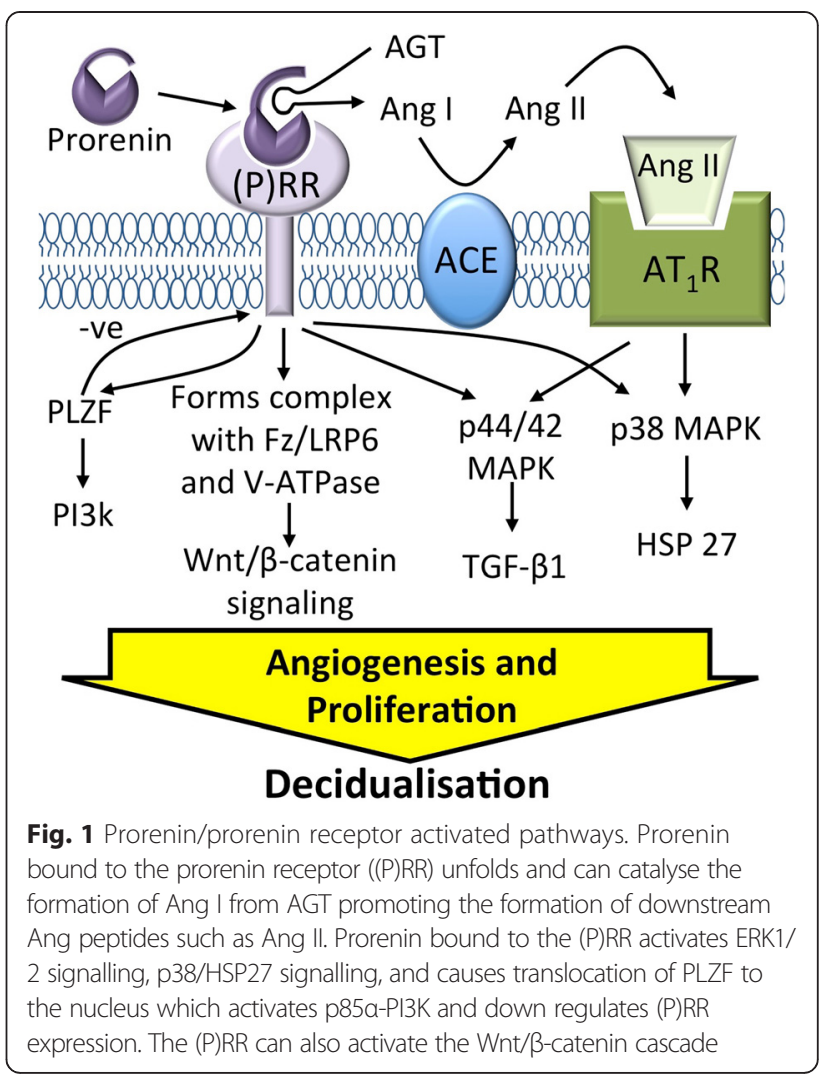

be decidualised in vitro $[13,14]$. Decidualisation is induced by incubation for $9-10$ days in medium containing a mixture of medroxyprogesterone acetate (MPA), 17 $\beta$ estradiol $\left(\mathrm{E}_{2}\right)$, and cyclic AMP (MPA-mix) [14]. HESCs are morphologically transformed from a fibroblast like appearance to large pale glycogen containing cells typical of decidual stromal cells. Furthermore they express prolactin, insulin-like growth factor binding protein-1 (IGFBP-1) and the cytoskeleton protein, desmin when induced to decidualise with MPA-mix [14]. Demethylation of HESCs by 5 -aza-2'deoxycytidine (AZA) also induces some of the characteristics of decidualisation [15].

Because there are high levels of expression of RAS genes in late gestation decidua, and there is cyclical expression of RAS proteins in human endometrium, we carried out experiments to find out if the genes of the RAS pathways (Fig. 1) are expressed in this endometrial stromal cell line, whether or not their expression is upregulated when decidualisation is induced, and whether this is mimicked by global demethylation using AZA. We also wanted to find out to what extent the effects of the decidualising 'cocktail' on RAS genes depend on $E_{2}$ and MPA independent of any effects of cAMP. Finally we compared the effects of AZA and MPA-mix on the expression of a number of genes and proteins known to stimulated by the prorenin/prorenin receptor interaction and the RAS cascade. 


\section{Methods \\ Cell Culture}

The hTERT immortalised HESCs (which originated from Dr Charles J Lockwood, Yale University School of Medicine, New Haven, CT, and were a gift from Dr. Peter A.W. Rogers, Monash University) were cultured in Dulbecco's modified Eagle's medium and Ham's F-12 (DMEM/F12) medium (Invitrogen Life Technologies, Auckland, New Zealand), which was supplemented with $10 \%$ heat inactivated fetal bovine serum (Gibco, Invitrogen Life Technologies), $100 \mathrm{mg} / \mathrm{ml}$ streptomycin, $100 \mathrm{U} /$ $\mathrm{ml}$ penicillin (Invitrogen Life Technologies) and $2 \mathrm{mM}$ glutamax (Invitrogen Life Technologies), and cultured at $37{ }^{\circ} \mathrm{C}$ in a humidified $5 \% \mathrm{CO}_{2}$ incubator. Cells were detached by trypsinization ( $1 \mathrm{x}$ trypsin-EDTA, Invitrogen Life Technologies).

A mixture of medroxyprogesterone acetate (MPA), $17 \beta$-estradiol $\left(\mathrm{E}_{2}\right)$ and dibutyryl cAMP (MPA-mix), in $2 \%$ FBS-supplemented media [16] or 5-aza-2'-deoxycytidine (AZA; Sigma-Aldrich, St Louis, MO, USA) which we know decidualises HESCs, was used [13-15]. In a second series of experiments, an additional treatment with $1 \mu \mathrm{M}$ MPA plus $10 \mathrm{nM} 17 \beta$-estradiol but without cAMP was included. The MPA-mix used published concentrations for decidualising endometrial stromal cells [17]. These were $1 \mu \mathrm{M}$ MPA (Calbiochem, SigmaAldrich), $10 \mathrm{nM}$ 17 $\beta$-estradiol (Sigma-Aldrich) and $0.5 \mathrm{mM}$ cAMP (Sigma-Aldrich) [16]. The AZA concentration was $15 \mu \mathrm{M}$, which had been determined by optimization on HESCs by semi-quantitative PCR of prolactin gene expression [15]. A three-way experiment was performed by treating HESCs with AZA or MPAmix or the control (0.01\% dimethylsulfoxide, DMSO, final concentration).

HESCs were seeded in six-well plates so that $60 \%$ confluent cells were treated the following day. The plates were incubated in a humidified incubator at $37{ }^{\circ} \mathrm{C}, 5 \%$ $\mathrm{CO}_{2}$, and the treatments were renewed with a change of media every 2 days, for 10 days. Total RNA was extracted from HESC cells using TRIZol ${ }^{\circledR}$ (Invitrogen Life Technologies) according to the manufacturer's instructions, with the addition of an extra chloroform step. Total RNA concentrations were determined by NanoDrop analysis (ND-1000 Spectrophotometer, Thermo Scientific, MA). Total RNA of each sample $(1 \mu \mathrm{g})$ was DNase treated and converted to cDNA for real-time quantitative PCR (qPCR) using SuperScript III and random hexamers (Invitrogen Life Technologies).

In a second series of experiments, the HESCs were treated similarly, but there were 4 treatments (control, $\mathrm{E}_{2}+\mathrm{MPA}$ alone, AZA and MPA-mix). In these experiments, media was collected after 2 days incubation and at 10 days when cells were recovered and stored at $-80{ }^{\circ} \mathrm{C}$ for determination of prorenin protein.
Real-time quantitative reverse transcriptase polymerase chain reaction (qPCR)

qPCR was performed in an Applied Biosystems 7500 Real Time PCR System using SYBR Green for detection. Each reaction contained cDNA reversed transcribed from $10 \mathrm{ng}$ total RNA, SYBR Green PCR master mix (Applied Biosystems, Carlsbad, CA), RAS primers that we have described previously [3, 18, 19]. The expression of RAS genes: angiotensinogen (AGT), prorenin $(R E N)$, (pro)renin receptor $(A T P 6 A P 2)$, angiotensin converting enzyme $(A C E 1)$ and $\mathrm{AT}_{1} \mathrm{R}(A G T R 1)$ were examined. In addition, VEGFA , PLZF, TGFB1, PIK3R1 and SERPINE1 mRNA levels were also measured. Primers for these genes are described in Table 1. Messenger RNA relative abundance was calculated as described previously, using the $2^{-}$ $\Delta \Delta \mathrm{CT}$ method, abundance is expressed relative to both $\beta$ actin $(A C T B)$ mRNA and a calibrator sample (pooled term decidua for experiment 1 , and a term placental sample collected at elective Caesarean section for experiment 2). Therefore, all gene expression data is expressed as a fold change relative to its respective control group.

\section{Measurement of prorenin, VEGF, PAI-1 and TGF- $\beta 1$ proteins by ELISA}

Prorenin levels in culture media collected every 2 days and measured at 2 days and 10 days using the Human Prorenin ELISA kit (Molecular Innovations Inc; Novi, MI) according to the manufacturer's instructions. Prorenin in each sample was captured by an antibody immobilised onto the surface of each well of the plate. A primary antibody specific for prorenin was then applied and the unbound fraction removed by washings. For subsequent detection by means of colour development, a secondary antibody conjugated to horseradish peroxidase was used, followed by 3,3,5,5 - tetramethylbenzidine (TMB) substrate. After termination of the reaction with $4 \mathrm{M}$ sulphuric acid, optical density was read at $450 \mathrm{~nm}$. In our laboratory $1 \mathrm{ng} / \mathrm{mL}$ amniotic fluid prorenin measured using this technique generated $116 \mathrm{ng} /$ $\mathrm{h} / \mathrm{mL}$ of Ang I at $37{ }^{\circ} \mathrm{C}$ from angiotensinogen present in nephrectomized sheep plasma used as the source of angiotensinogen substrate. All samples were assayed on one ELISA plate. Therefore there was no inter-assay variability. Intra-assay coefficient of variation was $7.3 \%$.

VEGF, PAI-1 and TGF- $\beta 1$ concentrations in culture media was measured using the Human VEGF, PAI-1 or TGF- $\beta 1$ Duoset ELISA kits, respectively (all from R\&D systems, Minneapolis, MN) according to the manufacturer's instructions. The protein of interest in each sample was captured by an antibody immobilised onto the surface of each well of the plate. For subsequent detection by means of colour development, a secondary antibody conjugated to horseradish peroxidase was used. Color reagent A (Hydrogen peroxide) and B (3,3,5,5tetramethylbenzidine (TMB) substrate) were mixed in 
Table 1 Primers used in real time PCR

\begin{tabular}{|c|c|c|c|c|}
\hline Gene & GenBank Accession \# & Primer Sequence $\left(5^{\prime}-3^{\prime}\right)$ & Conc'n (nM) & Annealing temp $\left({ }^{\circ} \mathrm{C}\right)$ \\
\hline \multirow[t]{2}{*}{ PLZF } & NM_000789 & F:TAGGGTGCACACAGGTGAGA & 200 & 60 \\
\hline & & R:GTGCAGATGGTGCACTGGTA & & \\
\hline \multirow[t]{2}{*}{ PIK3R1 } & NM_181523 & F:CGGATCTTGCAGAGCAGTTT & 600 & 75 \\
\hline & & R: AGGTTGCTGGAGCTCTGTGT & & \\
\hline \multirow[t]{2}{*}{ VEGF } & M32977 & F:CTACCTCCACCATGCCAAGT & 400 & 75 \\
\hline & & R: GCAGTAGCTGCGCTGATAGA & & \\
\hline \multirow[t]{2}{*}{ SERPINE1 } & P05121 & F:TCTGTGTCACCGTATCTCA & 200 & 75 \\
\hline & & R: GCTCCGTCACGCTGGATGTC & & \\
\hline \multirow[t]{2}{*}{ TGFB1 } & P01137 & F:GAACTCATTCAGTCACCATAGCAACACTCT & 400 & 70 \\
\hline & & R:TCTCTGGGCTTGTTTCCTCACCTTIA & & \\
\hline
\end{tabular}

$F$, forward; $R$, reverse

equal volumes and added to each well. After termination of the reaction with Stop Solution (2 N sulphuric acid), optical density was read at $450 \mathrm{~nm}$. TGF- $\beta 1$ samples were activated in order to measure total TGF- $\beta 1$. Each $100 \mu \mathrm{L}$ sample was incubated with $20 \mu \mathrm{L} 1 \mathrm{~N} \mathrm{HCL}$ for 10 minutes at room temperature, after which the reaction was stopped with $20 \mu \mathrm{L}$ of $1.2 \mathrm{~N} \mathrm{NaOH} / 0.5$ HEPES. The samples were assayed immediately after activation. The intra-plate coefficient of variation was $7.6 \%$, $3.8 \%$ and $6.9 \%$ for VEGF, PAI-1 and TGF- $\beta 1$ respectively. Each assay was run on one plate so there was no interassay coefficient of variation.

\section{Data Analysis}

Each experiment was carried out three times in triplicate so each experiment had a vehicle control and a treated set. Data from all 3 experimental sets were combined and univariate analysis with treatments and experiment number as fixed factors carried out using SPSS v21. Tukey's test was applied to correct for multiple corrections. Significance was set at $5 \%$.

\section{Results}

\section{Effects of treatments on genes of the prorenin/prorenin} receptor angiotensin system

Both series of experiments showed consistent results; measurable levels of REN, AGT, ATP6AP2 and ACE mRNA were found in untreated HESC samples. AGTR1 levels were low, and low to undetectable results were obtained for $A C E 2$.

In both series of experiments treatment with MPAmix and AZA were associated with upregulation of REN relative to vehicle treated controls $(\mathrm{P}<0.008$, Fig. 2a) and in the second experiment the increase in AGT mRNA abundance was also significant with MPA-mix treatment (Fig. 2b). MPA-mix caused a significantly greater increase in REN mRNA than did AZA treatment $(\mathrm{P}<0.007$, Fig. 2a) in both experiments. AZA treatment was also associated with a $400 \%$ and $170 \%$ increase in ACE mRNA relative to vehicle treated controls $(\mathrm{P}=0.026$ and $\mathrm{P}<0.08$ respectively). In the second experiment, this increase was significant compared with effects seen in $E_{2}$ + MPA treated cells $(\mathrm{P}=0.02)$. MPA-mix had no effect on $A C E$ expression. There were no other effects of either MPA-mix or AZA treatment on the other RAS genes studied in this experiment.

In the second series of experiments, prorenin protein was measured in media of untreated controls at 2 and 10 days incubation. Decidualisation with MPA-mix was associated with significantly greater levels of prorenin at 10 days but not at 2 days (Fig. 2c and d). Comparison of Fig. 2c and d show that prorenin levels in 10 day samples were lower than the concomitant 2 day samples in control and $E_{2}+$ MPA samples $(P<0.001)$ but were about the same in MPA-mix and AZA treated samples.

Effects of treatments on genes and proteins known to be responsive to stimulation by the prorenin receptor/ prorenin angiotensin system

The effects of the 3 treatments on the expression of VEGF and PAI-1 (SERPINE1) are shown in Fig. 3. Both $\mathrm{E}_{2}+\mathrm{MPA}$ alone and MPA-mix stimulated expression of SERPINE1 in HESCs $(\mathrm{P}<0.001$, Fig. 3a). PAI- 1 protein levels were increased in supernatants from both $E_{2}+$ MPA and MPA-mix treated HESCs $(\mathrm{P}<0.001)$ compared with control and AZA treated samples (Fig. 3c). PAI-1 protein levels were higher in $\mathrm{E}_{2}+\mathrm{MPA}$ treated HESCs compared with MPA-mix treated HESCs $(\mathrm{P}<0.006$, Fig. 3c). AZA treatment had no effect on SERPINE1 mRNA abundance nor on PAI-1 protein levels.

MPA-mix stimulated the expression of VEGF A (compared with all other treatments $\mathrm{P}<0.001$, Fig. $3 \mathrm{~b}$ ). VEGF protein levels were also increased in supernatant from HESC treated with MPA-mix compared with all other treatments $(\mathrm{P}<0.04$, Fig. $3 \mathrm{~d})$. AZA treatment had no effect on $V E G F A$ expression or VEGF protein levels. 
A

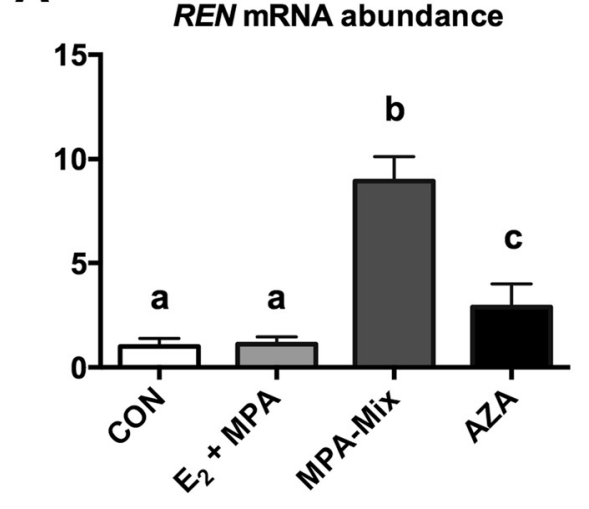

C

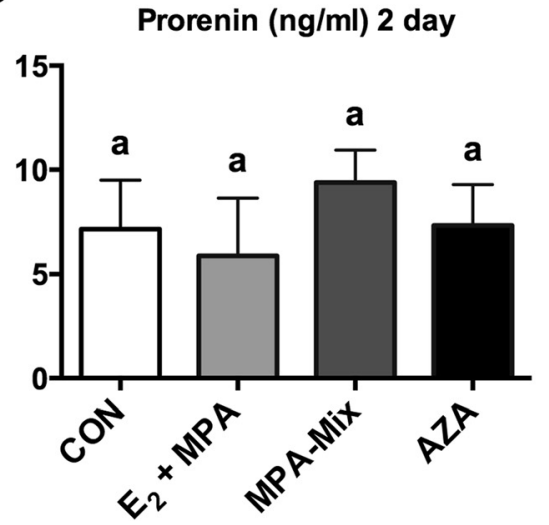

B

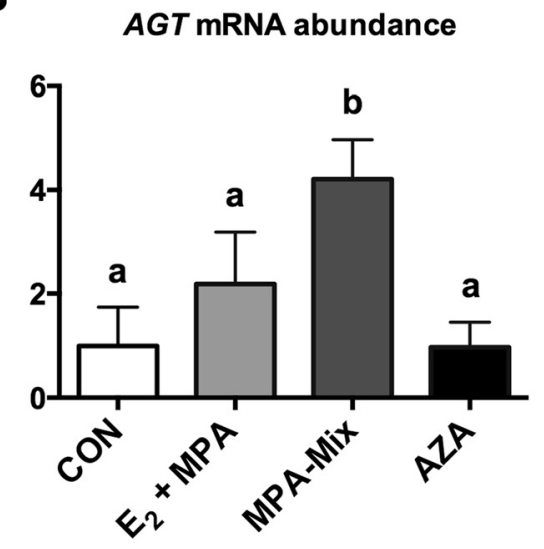

D

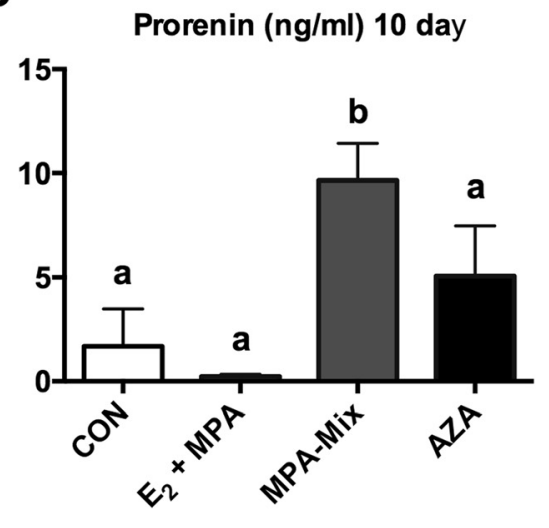

Fig. 2 Effects of $E_{2}+M P A, M P A-m i x$ and AZA on the renin angiotensin system in HESCs. a REN mRNA levels were significantly higher in HESCs treated with MPA-mix compared with all other treatments $(P<0.0001)$. REN mRNA expression was also increased in AZA treated HESCs compared with control and $E_{2}+$ MPA treated cells ( $<<0.008$ and $P<0.014$, respectively). $\mathbf{b}$ AGT mRNA levels were significantly increased in MPA-mix treated cells compared with all other treatments (all $\mathrm{P}<0.02)$. c Prorenin protein $(\mathrm{ng} / \mathrm{ml}$ ) secreted into media at 2 days. There was no difference between CON and treatments but MPA-mix had significantly more prorenin than $E_{2}+$ MPA alone $(P=0.045)$. $\mathbf{d}$ Prorenin protein (ng/ml) secreted into media at 10 days. Prorenin levels were significantly increased in the supernatant at 10 days in MPA-mix treated samples compared with all other treatments $(P<0.022)$. AZA treatment was associated with increased prorenin levels in supernatant compared with $E_{2}+M P A$ alone $(P<0.015)$. Different superscripts denote differences between groups. CON, treated with vehicle alone; $E_{2}+M P A$, treatment with 10 nM $17 \beta$-estradiol ( $\left.E_{2}\right)$ and $1 \mu \mathrm{M}$ medroxyprogesterone acetate (MPA); $\mathrm{E}_{2}+\mathrm{MPA}+\mathrm{CAMP}$, treatment as for $\mathrm{E}_{2}+\mathrm{MPA}$ plus $0.5 \mathrm{mM}$ CAMP

There was no effect of any treatment on the expression of TGFB1 (data not shown). There was also no effect on total, active and latent TGF- $\beta 1$ protein levels.

Effects of treatments on genes known to interact with the prorenin/prorenin receptor system but which have effects independent of formation of Ang peptides

Two genes were studied; PLZF and its downstream target p85 $\alpha$-PI3kinase. PLZF mRNA were not detected in control or AZA treated cells but were expressed in both $\mathrm{E}_{2}+\mathrm{MPA}$ and MPA-mix treated HESCs, although the effect of $E_{2}+M P A$ treatment was significantly less than that seen with MPA-mix (Fig. 4a). AZA treatment had no effect. p85 $\alpha$-PI3kinase (PIK3R1) mRNA expression was significantly increased in MPA-mix treated HESCs compared with those treated with $\mathrm{E}_{2}+\mathrm{MPA}$ alone $(\mathrm{P}=$
0.007) although it was not significantly greater then control $(\mathrm{P}<0.07)$.

\section{Discussion}

The transformation of a stromal endometrial cell to a decidual cell (decidualisation) occurs late in the menstrual cycle under the influence of the hormones estrogen and progesterone. The subsequent accumulation of intracellular cAMP triggers the transformation from a fibroblast phenotype to an inflammatory and finally a secretory phenotype [20]. Previous reports have demonstrated that this can be mimicked in vitro as progesterone, either alone or in combination with estradiol, stimulates intracellular cAMP accumulation and decidualisation of human endometrial stromal cells in culture [21, 22]. We have shown that the morphological and molecular changes (increased 
A

PAI-1 mRNA abundance

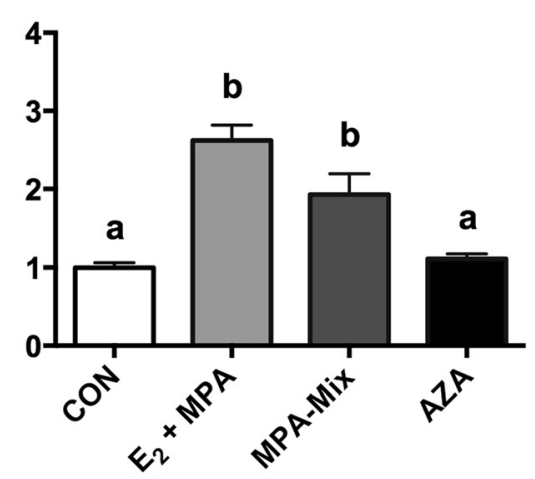

C
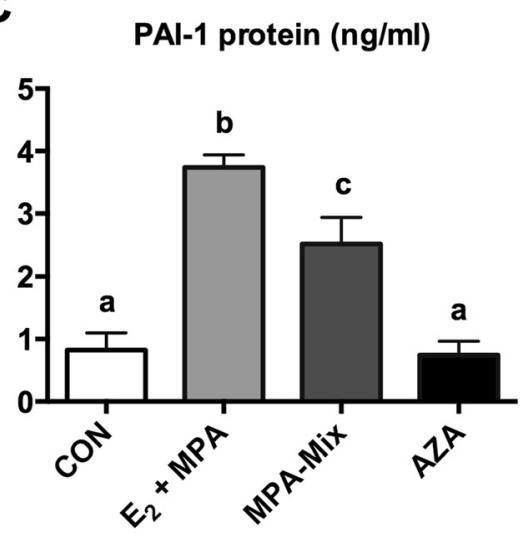

B
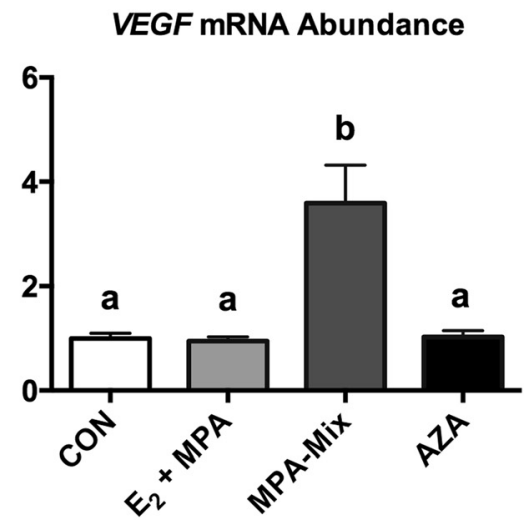

D

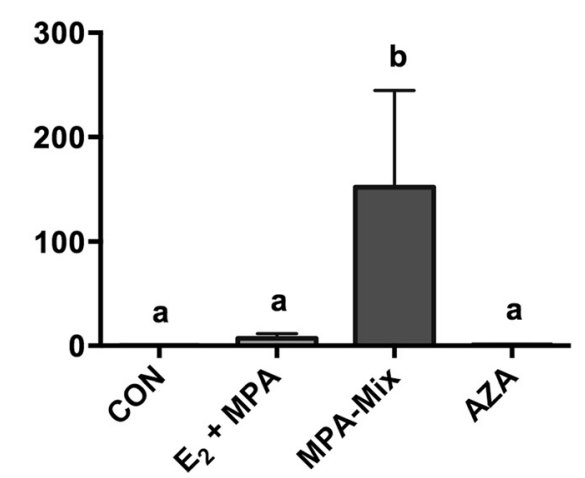

Fig. 3 Effects on the expression of genes and proteins known to be responsive to stimulation by the prorenin receptor/prorenin angiotensin system. $\mathbf{a}$ and $\mathbf{c}$ Relative mRNA abundance of PAI-1 (SERPINE1) and its protein. Both $\mathrm{E}_{2}+$ MPA alone treatment and MPA-mix treatment stimulated expression of PAI-1 (SERPINE1) in HESCS $(P<0.001$ ). AZA had no effect. $\mathbf{b}$ and $\mathbf{d}$ MPA-mix treatment stimulated the expression of VEGF Aand its protein compared with all other treatments (all $P<0.001$ ). Different superscripts denote differences between groups. CON, treated with vehicle alone; $E_{2}+M P A$, treatment with $10 \mathrm{nM} 17 \beta$-estradiol $\left(E_{2}\right)$ and $1 \mu M$ medroxyprogesterone acetate $(M P A) ; E_{2}+M P A+c A M P, E_{2}+M P A+c A M P$, treatment as for $E_{2}+$ MPA plus 0.5 mM cAMP

A

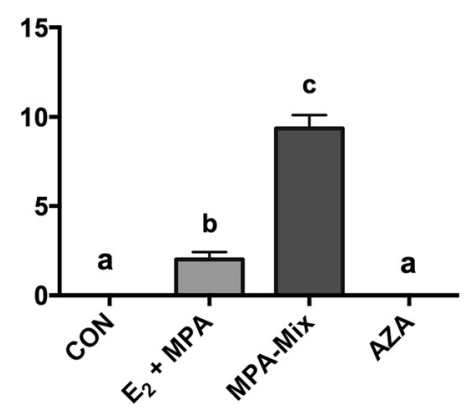

B

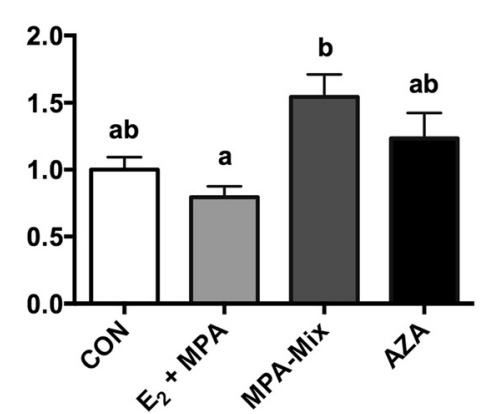

Fig. 4 Effect of $E_{2}+M P A, M P A-m i x ~\left(E_{2}, M P A+C A M P\right)$ and $A Z A$ on the expression of genes known to interact with prorenin/prorenin receptor that are independent of Ang II. a PLZF (Promyelocytic leukaemic zinc finger protein) mRNA was not detected in CON or AZA treated cells but was present in $E_{2}+$ MPA and MPA-mix treated cells $(P<0.001$ for both treatments compared with CON and AZA). Expression of PLZF in MPA-mix treated cells was much greater than in $E_{2}+$ MPA treated cells $(P<0.001)$. b p85a-PI3kinase (PIK3R1) mRNA expression was significantly increased in MPA-mix treated HESCs compared with those treated with $E_{2}+$ MPA alone $(P=0.007)$ although it was not greater then control $(P<0.07)$. Different superscripts denote differences between groups. CON, treated with vehicle alone; $E_{2}+M P A$, treatment with $10 \mathrm{nM} 17 \beta-e s t r a d i o l\left(E_{2}\right)$ and $1 \mu \mathrm{M}$ medroxyprogesterone acetate (MPA); $E_{2}+M P A+C A M P$, treatment as for $E_{2}+$ MPA plus $0.5 \mathrm{mM}$ cAMP 
expression of FOXO1, PRL and IGFBP-1) that characterise decidualisation are also recreated in human endometrial stromal cells (HESCs) in vitro using MPA-mix [14] and to some extent, by AZA treatment [15]. Overall the stimulatory effects of AZA treatment on the key markers of decidualisation, namely prolactin and IGFBP-1 are much less than effects of MPA mix [14]. Similarly, in this study, the effects of AZA on the renin-angiotensin system and the downstream signalling genes is much less than the effects seen with decidualisation induced by MPA mix.

Despite the presence of renin-angiotensin system proteins in human endometrium, their cyclical expression [2], and the fact that the decidua is the major intrauterine tissue producing prorenin in late gestation [3-5], the effects of decidualisation on expression of the endometrial RAS have not previously been described. In this study we characterized the effects of decidualisation of HESCs on its RAS.

HESCs express ATP6AP2, REN, AGT, ACE and low levels of AGTR1 and ACE2. When treated with MPA-mix, expression of REN was markedly increased, as was the secretion of prorenin protein (Fig. 2). The effect of MPA-mix on prorenin protein was unlikely due to increased cell proliferation, as we have previously demonstrated that treatment with MPA-mix or AZA significantly inhibits cell proliferation in HESCs in vitro [23]. Expression of AGT was also increased in the second experiment (in the first experiment the increase in AGT did not reach significance). Global demethylation with 5-aza-2'deoxycytidine, which induces decidualisation [15], was also associated with upregulation of REN but to a lesser extent than that seen with MPA-mix (Fig. 2). Treatment with AZA had no effect on $A G T$ in either experiment but did cause upregulation of $A C E$ expression in both experiments. Interestingly, the amount of prorenin in the supernatant was less in control and $E_{2}+$ MPA samples at 10 days compared with 2 days (Fig. 2c and d). This is most likely due to a residual stimulatory effect of fetal bovine serum on prorenin secretion as the cells are cultured in media containing $10 \%$ FBS prior to the experiment and in $2 \%$ FBS during the experiment itself. As the media were changed every 2 days the effect of the experimental treatments, as opposed to FBS can be seen more effectively at 10 days. $\mathrm{E}_{2}+$ MPA treatment without cAMP had no effect on $R E N, A G T$ or $A C E$ mRNA abundance nor on prorenin protein levels.

In previous studies we have shown that within the intrauterine tissues prorenin expression is highest in the decidua in late gestation [3], that decidual explants grown ex vivo for $48 \mathrm{~h}$ secrete prorenin, and that both decidual expression of REN and prorenin protein levels are highest if the fetus is female $[4,5]$. The present studies show that decidual prorenin secretion, which is constitutive, is switched on when stromal cells are decidualised. Thus stromal cells express the renin gene and secrete prorenin, independent of the presence of glandular cells which also contain renin [2] as do placental villous cells which are also responsive to cAMP in terms of REN expression and prorenin secretion $[24,25]$.

Increased expression of prorenin and its secretion in response to MPA-mix, i.e. when decidualisation occurs, is not surprising since cAMP plays a pivotal role in inducing the phenotypic changes that result in transformation of the fibroblast like stromal cell to a secretory decidual cell [20]. cAMP also regulates REN expression. Maximal cAMP induction of REN depends on a cAMP response element (CRE) in the proximal promoter region that binds cAMP response element binding protein (CREB) as well as a factor interacting with a Pit-1 motif in the REN gene [26]. The lack of response to $\mathrm{E}_{2}+\mathrm{MPA}$ alone in HESCs suggests that these two agents alone did not stimulate cAMP production to an extent sufficient to upregulate $R E N$ expression (Fig. 2).

CAMP, via a CRE in the AGT gene, also stimulates expression and secretion of AGT by adipose tissue [27]. As well, AGT is stimulated by estradiol through a cis-acting DNA element situated between the TATA box and the start of the transcription site [28]. AGT expression in a number of extra-hepatic tissues is sensitive to $E_{2}$ [29]. It is, therefore not surprising that $A G T$ was also stimulated by decidualisation of HESCs. The effect of MPA-mix on $A G T$ was however not as consistent as its effect on REN, in that the increase in $A G T$ mRNA did not reach statistical significance in the first set of experiments; it was only evident in the second set.

In a previous study, Logan et al. showed that global demethylation of HESCs with 5-aza-2'deoxycytidine caused down regulation of DNA methyltransferases (DNMTs) and an upregulation of those genes that characterize decidualisation e.g. prolactin, IGFBP-1 and FOX01 [15]. Therefore we studied the effects of AZA-induced decidualisation on expression of HESC RAS genes. In both sets of experiments AZA treatment caused significant upregulation of REN expression. Levels of prorenin protein secreted by AZA treated HESCs were greater at 10 days than those measured in cells treated with $E_{2}+$ MPA alone but they were not different from control levels. The difference in prorenin protein levels between these two treatments might be accounted for by the decline in prorenin secretion by $E_{2}+$ MPA treated cells.

AZA treatment, unlike treatment with MPA-mix, also appeared to affect the expression of $A C E$. In the first set of experiments $A C E$ mRNA was greater in AZA treated HESCs than in vehicle treated controls, while in the second series of experiments $A C E$ mRNA abundance was no different from vehicle treated controls but was greater than that measured in $\mathrm{E}_{2}+\mathrm{MPA}$ treated cells. $A C E$ is one of the few genes in the RAS pathway (ATP6AP2, ACE and AGTR1) that have an abundance of CpG islands near the 
promoter region of the gene. Therefore one might have expected that demethylation by AZA would have upregulated expression of $A C E$. The effect was marginal however and AZA treatment had no effect on ATP6AP2 nor on AGTR1 expression.

Prorenin is catalytically inactive in vivo unless it is unfolded by low $\mathrm{pH}$ treatment or cold, bound to its prorenin receptor or its pro-segment is removed by proteases $[6-8,30]$. Assembly of V-ATPase depends on the (P)RR; the absence of the (P)RR leads to decreased expression of Vo subunits of V-ATPase and de-acidification of intracellular vesicles [9]. Since prorenin is susceptible to unfolding in the presence of a low $\mathrm{pH}$ [6] it is tempting to suggest that the intimate association between the (P)RR and VATPase exposes secreted prorenin to a low pH milieu which unfolds the pro-segment perhaps facilitating binding to the receptor or access by AGT to the catalytic site.

Independent of the RAS, binding of prorenin to the (P)RR activates ERK1/2 pathways stimulating TGF- $\beta 1$ and PAI-1 production in mesangial cells in culture; it also stimulates proliferation of these cells [31].

Prorenin binding to the cardiac prorenin receptor stimulates HSP27/p38 MAPK [10]. When prorenin alone is applied to cardiac myocytes it does not stimulate ERK $1 / 2$, AGT is also required. In the heart, it is Ang II acting via its $\mathrm{AT}_{1} \mathrm{R}$ that stimulates PAI- 1 production by cardiac myocytes [10,32]. Ang II acting via $\mathrm{AT}_{1} \mathrm{R}$ also stimulates angiogenesis possibly because it stabilizes HIF-1a leading to increased expression of VEGF [33].

Finally binding of prorenin to the prorenin receptor causes translocation of the transcription factor, PLZF, to the nucleus where it stimulates expression of p85 $\alpha$ PI3kinase, causing cell proliferation, and down regulates ATP6AP2 expression (Fig. 1) [11].

Therefore we examined the expression of 3 downstream pathways that could be activated by the prorenin/prorenin receptor angiotensin system. VEGF $A$ and SERPINE1 expression were increased when HESCs were decidualised with MPA-mix but not when they were treated with AZA. SERPINE1expression was also increased when cells were treated with $E_{2}+$ MPA alone. Levels of VEGF and PAI-1 protein reflected the effects of treatment on expression of their genes (Fig. 3). Rather surprisingly no treatment affected the expression of TGFB1 and levels of either latent or total TGF- $\beta 1$ protein.

$P L Z F$ was not detected in vehicle treated control cells nor in AZA treated cells but was expressed in cells treated with $E_{2}+$ MPA and further enhanced by treating with MPA-mix. It is not surprising that PLZF was expressed in both $E_{2}+$ MPA and MPA-mix treated cells because progesterone is known to stimulate the expression of this transcription factor [34]. The effects, if any, of high levels of expression of PLZF and increased secretion of prorenin on p85 $\alpha$-PI3kinase expression were marginal in that p85 $\alpha$-PI3kinase mRNA levels were only increased in MPA-mix relative to $E_{2}+$ MPA alone, and not significantly increased compared with control values. We have not however examined phosphorylation of p85 $\alpha$-PI3kinase in this study, which could provide further insight into the possible activation of this pathway during decidualisation.

\section{Conclusion}

In conclusion, HESCs express ATP6AP2, REN, AGT and $A C E$. When treated for 10 days with a cocktail containing cAMP, $E_{2}$ and MPA to induce decidualisation, there is upregulation of REN and $A G T$ expression and prorenin secretion. Global demethylation of HESC with AZA, which mimics decidualisation [15], also resulted in increased expression of REN. Levels of expression of other genes that have been shown to be influenced by the activity of the prorenin receptor/prorenin angiotensin system, namely VEGF $A$ and SERPINE1 were also increased but other effects that can also be attributed to Ang II independent pathways, i.e. stimulation of TGFB1 and PIK3R1,were absent or minimal. High levels of expression of $R E N$ and increased secretion of prorenin protein when HESCs were decidualised suggests that this system plays a role in regulating cell function in decidua and adjacent tissues throughout pregnancy. In particular, the activation of expression of VEGF and its secretion in association with upregulation of the RAS in HESCs suggests that decidualisation of the endometrial stromal RAS could play a critical role in inducing increased vascularity of the decidua so ensuring an adequate blood flow to the placenta.

\section{Abbreviations}

ACE: Angiotensin-converting enzyme; ACE2: Angiotensin-converting enzyme 2; ACTB: $\beta$-actin gene; AGT: Angiotensinogen; AT ${ }_{1} R / A G T R 1$ : Angiotensin II type 1 receptor; Ang: Angiotensin; AT R/AGTR2: Angiotensin II type 2 receptor; Ang: Angiotensin; AZA: 5-aza-2'deoxycytidine; CAMP: Cyclic AMP; $E_{2}: 17 \beta$-estradiol; FBS: Fetal bovine serum; HESC: Human endometrial stromal cell line; MPA: Medroxy-progesterone acetate; PAI-1/SERPINE1: Plasminogen activator inhibitor-1; PIK3R1: Phosphor-inositol-3 kinase; PLZF: Promyelocytic zinc finger protein; (P)RR/ATP6AP2: (Pro)renin receptor; RAS: Renin

angiotensin system; REN: (pro)renin; TGF- $\beta 1 /$ TGFB1: Transforming growth factor- $\beta 1$; V-ATPase: Vacuolar $\mathrm{H}^{+}$-ATPase; VEGF: Vascular endothelial growth factor.

\section{Competing interests}

None of the authors have any competing interests to declare.

\section{Author's contributions}

E. R. Lumbers was responsible for the design of these experiments analysis of data and writing the manuscript. K. G. Pringle managed the project and contributed to manuscript preparation, P. C. Logan and M. D. Mitchell developed the techniques for decidualising HESCs and carried out cell cultures. S. J. Delforce and Y. Wang carried out real time PCR of RAS genes and analysed data. C. Corbisier de Meaultsart carried out ELISAs and analysed data. All authors read and approved the final manuscript.

\section{Acknowledgements}

This research was funded by a National Health and Medical Research Council of Australia (NHMRC) project grant GNT517406 awarded to ERL. 


\section{Author details}

'School of Biomedical Sciences and Pharmacy, University of Newcastle, Newcastle, Australia and Mothers and Babies Research Centre, Hunter Medical Research Institute, Level 3 East, 1 Kookaburra Circuit, New Lambton Heights, NSW 2305, Australia. ${ }^{2}$ The Liggins Institute, University of Auckland, Auckland, New Zealand. ${ }^{3}$ University of Queensland Centre for Clinical Research, University of QLD, St Lucia, QLD, Australia. ${ }^{4}$ Present address: Reproductive Sciences, Department of Obstetrics and Gynecology, School of Medicine, University of California San Francisco, San Francisco, USA.

Received: 17 August 2015 Accepted: 19 November 2015

Published online: 25 November 2015

\section{References}

1. Li XF, Ahmed A. Expression of angiotensin II and its receptor subtypes in endometrial hyperplasia: a possible role in dysfunctional menstruation. Lab Invest. 1996;75(2):137-45.

2. Li XF, Ahmed A. Compartmentalization and cyclic variation of immunoreactivity of renin and angiotensin converting enzyme in human endometrium throughout the menstrual cycle. Hum Reprod. 1997;12(12):2804-9.

3. Pringle KG, Zakar T, Yates D, Mitchell CM, Hirst JJ, Lumbers ER. Molecular evidence of a (pro)renin/ (pro)renin receptor system in human intrauterine tissues in pregnancy and its association with PGHS-2. J Renin Angiotensin Aldosterone Syst. 2011;12(3):304-10.

4. Wang $Y$, Lumbers ER, Sykes SD, Pringle KG. Regulation of the ReninAngiotensin System Pathways in the Human Decidua. Reprod Sci. 2014;22(7):72-865

5. Wang Y, Pringle KG, Sykes SD, Marques FZ, Morris BJ, Zakar T, et al. Fetal sex affects expression of renin-angiotensin system components in term human decidua. Endocrinology. 2012;153(1):462-8.

6. Lumbers ER. Activation of renin in human amniotic fluid by low $\mathrm{pH}$. Enzymologia. 1971;40(6):329-36

7. Pitarresi TM, Rubattu S, Heinrikson R, Sealey JE. Reversible cryoactivation of recombinant human prorenin. J Biol Chem. 1992;267(17):11753-9.

8. Nguyen G, Delarue F, Burckle C, Bouzhir L, Giller T, Sraer JD. Pivotal role of the renin/prorenin receptor in angiotensin II production and cellular responses to renin. J Clin Invest. 2002;109(11):1417-27.

9. Kinouchi K, Ichihara A, Sano M, Sun-Wada GH, Wada Y, Kurauchi-Mito A, et al. The (pro)renin receptor/ATP6AP2 is essential for vacuolar $\mathrm{H}+-$ ATPase assembly in murine cardiomyocytes. Circ Res. 2010;107(1):30-4.

10. Saris JJ, T Hoen PA, Garrelds IM, Dekkers DH, Den Dunnen JT, Lamers JM. Prorenin induces intracellular signaling in cardiomyocytes independently of angiotensin II. Hypertension. 2006;48(4):564-71.

11. Schefe JH, Menk M, Reinemund J, Effertz K, Hobbs RM, Pandolfi PP, et al. A novel signal transduction cascade involving direct physical interaction of the renin/prorenin receptor with the transcription factor promyelocytic zinc finger protein. Circ Res. 2006;99(12):1355-66.

12. Krop M, Lu X, Danser AH, Meima ME. The (pro)renin receptor. A decade of research: what have we learned? Pflugers Arch. 2013;465(1):87-97.

13. Krikun G, Mor G, Alvero A, Guller S, Schatz F, Sapi E, et al. A novel immortalized human endometrial stromal cell line with normal progestational response. Endocrinology. 2004;145(5):2291-6.

14. Logan PC, Ponnampalam AP, Steiner M, Mitchell MD. Effect of cyclic AMP and estrogen/progesterone on the transcription of DNA methyltransferases during the decidualization of human endometrial stromal cells. Mol Hum Reprod. 2013;19(5):302-12.

15. Logan PC, Ponnampalam AP, Rahnama F, Lobie PE, Mitchell MD. The effect of DNA methylation inhibitor 5-Aza-2'-deoxycytidine on human endometrial stromal cells. Hum Reprod. 2010;25(11):2859-69.

16. Kasahara K, Takakura K, Takebayashi K, Kimura F, Nakanishi K, Noda Y. The role of human chorionic gonadotropin on decidualization of endometrial stromal cells in vitro. J Clin Endocrinol Metab. 2001;86(3):1281-6.

17. Tang B, Guller S, Gurpide E. Cyclic adenosine 3',5'-monophosphate induces prolactin expression in stromal cells isolated from human proliferative endometrium. Endocrinology. 1993;133(5):2197-203.

18. Marques FZ, Pringle KG, Conquest A, Hirst JJ, Markus MA, Sarris M, et al. Molecular characterization of renin-angiotensin system components in human intrauterine tissues and fetal membranes from vaginal delivery and cesarean section. Placenta. 2011;32(3):214-21.
19. Pringle KG, Tadros MA, Callister RJ, Lumbers ER. The expression and localization of the human placental prorenin/renin-angiotensin system throughout pregnancy: roles in trophoblast invasion and angiogenesis? Placenta. 2011;32(12):956-62.

20. Gellersen B, Brosens JJ. Cyclic decidualization of the human endometrium in reproductive health and failure. Endocr Rev. 2014;35(6):851-905.

21. Brar AK, Frank GR, Kessler CA, Cedars MI, Handwerger S. Progesteronedependent decidualization of the human endometrium is mediated by CAMP. Endocrine. 1997;6(3):301-7.

22. Brosens JJ, Hayashi N, White JO. Progesterone receptor regulates decidual prolactin expression in differentiating human endometrial stromal cells. Endocrinology. 1999;140(10):4809-20.

23. Logan PC, Steiner M, Ponnampalam AP, Mitchell MD. Cell cycle regulation of human endometrial stromal cells during decidualization. Reprod Sci. 2012;19(8):883-94.

24. Downing GJ, Poisner AM, Barnea ER. First-trimester villous placenta has high prorenin and active renin concentrations. Am J Obstet Gynecol. 1995;172(3):864-7.

25. Downing GJ, Yan B, Poisner AM. Beta-adrenoceptor activation-induced placental prorenin secretion is mediated by increased renin messenger RNA and protein synthesis. Mol Pharmacol. 1997;51(2):201-8.

26. Germain S, Konoshita T, Philippe J, Corvol P, Pinet F. Transcriptional induction of the human renin gene by cyclic AMP requires cyclic AMP response element-binding protein (CREB) and a factor binding a pituitaryspecific trans-acting factor (Pit-1) motif. Biochem J. 1996;316(Pt 1):107-13.

27. Serazin V, Dos Santos E, Morot M, Giudicelli Y. Human adipose angiotensinogen gene expression and secretion are stimulated by cyclic AMP via increased DNA cyclic AMP responsive element binding activity. Endocrine. 2004;25(2):97-104.

28. Yanai K, Nibu Y, Murakami K, Fukamizu A. A cis-acting DNA element located between TATA box and transcription initiation site is critical in response to regulatory sequences in human angiotensinogen gene. J Biol Chem. 1996;271(27):15981-6.

29. Klett C, Hellmann W, Hackenthal E, Ganten D. Modulation of tissue angiotensinogen gene expression by glucocorticoids, estrogens, and androgens in SHR and WKY rats. Clin Exp Hypertens. 1993;15(4):683-708.

30. Morris BJ, Lumbers ER. The activation of renin in human amniotic fluid by proteolytic enzymes. Biochim Biophys Acta. 1972;289(2):385-91.

31. Zhang J, Wu J, Gu C, Noble NA, Border WA, Huang Y. Receptor-mediated nonproteolytic activation of prorenin and induction of TGF-beta1 and PAl-1 expression in renal mesangial cells. Am J Physiol Renal Physiol. 2012;303(1):F11-20.

32. Huang Y, Noble NA, Zhang J, Xu C, Border WA. Renin-stimulated TGF-beta1 expression is regulated by a mitogen-activated protein kinase in mesangial cells. Kidney Int. 2007;72(1):45-52.

33. Liu C, Zhang JW, Hu L, Song YC, Zhou L, Fan Y, et al. Activation of the AT1R/HIF-1 alpha /ACE axis mediates angiotensin II-induced VEGF synthesis in mesenchymal stem cells. Biomed Res Int. 2014;2014:627380.

34. Fahnenstich J, Nandy A, Milde-Langosch K, Schneider-Merck T, Walther N, Gellersen B. Promyelocytic leukaemia zinc finger protein (PLZF) is a glucocorticoid- and progesterone-induced transcription factor in human endometrial stromal cells and myometrial smooth muscle cells. Mol Hum Reprod. 2003;9(10):611-23.

\section{Submit your next manuscript to BioMed Central and we will help you at every step:}

- We accept pre-submission inquiries

- Our selector tool helps you to find the most relevant journal

- We provide round the clock customer support

- Convenient online submission

- Thorough peer review

- Inclusion in PubMed and all major indexing services

- Maximum visibility for your research

Submit your manuscript at www.biomedcentral.com/submit 